\title{
INTERPOLATING OPERATORS IN NEST ALGEBRAS
}

\author{
M. ANOUSSIS
}

(Communicated by Paul S. Muhly)

\begin{abstract}
Given two families $\left\{x_{\alpha}\right\},\left\{y_{\alpha}\right\}$ of vectors and a nest $\mathscr{N}$ in a Hilbert space $H$, we provide a necessary and sufficient condition for the existence of an operator $T$ in the nest algebra satisfying $T x_{\alpha}=y_{\alpha}$ for every $\alpha$.
\end{abstract}

Given two families of vectors $\left\{x_{\alpha}\right\},\left\{y_{\alpha}\right\}$ in a Hilbert space, an interpolating operator is a bounded operator $T$ such that $T x_{\alpha}=y_{\alpha}$ for every $\alpha$. In [5] Lance gives a necessary and sufficient condition on two vectors $x$ and $y$ for the existence of an operator $T$ lying in a given nest algebra such that $T x=y$. Hopenwasser extended this result to the case of a CSL algebra [3]. Other questions about interpolating operators are investigated in [1, 4]. In this note we give a necessary and sufficient condition on two families $\left\{x_{\alpha}\right\},\left\{y_{\alpha}\right\}$ of vectors in a Hilbert space for the existence of an operator $T$ lying in a given nest algebra, such that $T x_{\alpha}=y_{\alpha}$ for every $\alpha$.

Throughout this note $H$ denotes a complex Hilbert space and $B(H)$ denotes the space of all bounded operators from $H$ to itself. By a nest we mean a complete totally ordered set of orthogonal projections. If $\mathscr{N}$ is a nest in $H$, then $\operatorname{Alg} \mathscr{N}$ is the set of operators $X$ in $B(H)$ satisfying $N^{\perp} X N=0$ for every $N$ in $\mathscr{N}$.

The main step in our proof is Proposition 3. We need two lemmas.

Lemma 1. Let $x_{1}, x_{2}, \ldots, x_{m}, y_{1}, y_{2}, \ldots, y_{m}$ be vectors in $H$. Let $V$ be the span of the $x_{i}$ 's, $i=1,2, \ldots, m$. Assume that there exists a real number $M>0$ such that:

$$
\left\|\sum_{i=1}^{m} c_{i} y_{i}\right\| \leq M \cdot\left\|\sum_{i=1}^{m} c_{i} x_{i}\right\|
$$

for every m-tuple $\left(c_{1}, c_{2}, \ldots, c_{m}\right)$ of complex numbers. Then there exists a linear operator $T: V \rightarrow H$ such that $T x_{i}=y_{i}$, for $i=1,2, \ldots, m$ and $\|T\| \leq M$.

Proof. We may assume that there exists an index $k, 1 \leq k \leq m$ such that the set $\left\{x_{1}, x_{2}, \ldots, x_{k}\right\}$ is a basis of $V$. Define $T: V \rightarrow H$ such that $T x_{i}=y_{i}$, $1 \leq i \leq k$. Then $T$ is a linear operator from $V$ to $H$, and by the condition of the lemma, it follows that $\|T\| \leq M$. We have to show that $T x_{i}=y_{i}$ for every

Received by the editors September 4, 1990.

1980 Mathematics Subject Classification (1985 Revision). Primary 47D25. 
$i, 1 \leq i \leq m$. Suppose, to the contrary, there exists $l, k<l \leq m$ such that $T x_{l} \neq y_{l}$. There are complex numbers $a_{i}, 1 \leq i \leq k$ such that $x_{l}=\sum_{i=1}^{k} a_{i} x_{i}$. Then,

$$
\left\|\sum_{i=1}^{k} a_{i} y_{i}-y_{l}\right\|=\left\|\sum_{i=1}^{k} a_{i} T x_{i}-y_{l}\right\|=\left\|T\left(\sum_{i=1}^{k} a_{i} x_{i}\right)-y_{l}\right\|=\left\|T x_{l}-y_{l}\right\| \neq 0,
$$

while $\left\|\sum_{i=1}^{k} a_{i} x_{i}-x_{l}\right\|=0$, contradicting the hypothesis.

Lemma 2. Let $A$ be a positive contraction in $B(H)$. We denote by $B$ the positive semidefinite hermitian form on $H$ defined by $B(x, y)=\langle x, A y\rangle$ for $x, y$ in $H$. Let $V$ be a finite-dimensional subspace of $H$. Then there exists $a$ closed subspace $V_{0}$ of $H$ such that:

(1) $V+V_{0}=H, V \cap V_{0}=\{0\}$;

(2) $B(x, y)=0$ for every $x$ in $V$ and $y$ in $V_{0}$.

Proof. Let $V_{1}=\{x \in H: B(x, y)=0, \forall y \in V\}=(A V)^{\perp}$. We show that $V+V_{1}=H$. Let $z$ be a vector in $\left(V+V_{1}\right)^{\perp}=V^{\perp} \cap V_{1}^{\perp}$. Since $V_{1}=(A V)^{\perp}$, which has finite codimension, $z$ is of the form $A v$ for some $v$ in $V$. We have:

$$
\langle v, A v\rangle=0 \Rightarrow A^{1 / 2} v=0 \Rightarrow A v=0 \Rightarrow z=0 .
$$

Let $V_{0}=V_{1} \ominus\left(V \cap V_{1}\right)$. We have:

$$
V+V_{0}=V+V_{1}=H, \quad V \cap V_{0}=\left(V \cap V_{1}\right) \cap V_{0}=\{0\} .
$$

It follows that the first condition of the lemma is satisfied. The second condition holds because $V_{0}$ is contained in $V_{1}$.

Proposition 3. Let $\mathscr{N}=\left\{0=N_{0}, N_{1}, N_{2}, \ldots, N_{k}=I\right\}$ be a finite nest in $H$. Let $x_{1}, x_{2}, \ldots, x_{m}, y_{1}, y_{2}, \ldots, y_{m}$ be vectors in $H$. Let $M>0$. The following are equivalent:

(1) There exists an operator $T$ in $\operatorname{Alg} \mathscr{N}$ such that $T x_{i}=y_{i}$, for $i=$ $1,2, \ldots, m$, and $\|T\| \leq M$.

(2) $\left\|N^{\perp}\left(\sum_{i=1}^{m} c_{i} y_{i}\right)\right\| \leq M \cdot\left\|N^{\perp}\left(\sum_{i=1}^{m} c_{i} x_{i}\right)\right\|$, for every m-tuple of complex numbers $\left(c_{1}, c_{2}, \ldots, c_{m}\right)$, and every projection $N$ in $\mathscr{N}$.

Proof. The implication (1) $\Rightarrow(2)$ is easy:

$$
\begin{aligned}
\left\|N^{\perp}\left(\sum_{i=1}^{m} a_{i} y_{i}\right)\right\| & =\left\|N^{\perp} T\left(\sum_{i=1}^{m} a_{i} x_{i}\right)\right\|=\left\|N^{\perp} T N^{\perp}\left(\sum_{i=1}^{m} a_{i} x_{i}\right)\right\| \\
& \leq\left\|N^{\perp} T\right\| \cdot\left\|N^{\perp}\left(\sum_{i=1}^{m} a_{i} x_{i}\right)\right\| \leq M \cdot\left\|N^{\perp}\left(\sum_{i=1}^{m} a_{i} x_{i}\right)\right\| .
\end{aligned}
$$

The converse is shown by induction on the length $k$ of the nest $\mathscr{N}$. We may assume that $M=1$.

First suppose that $k=1$. It follows from Lemma 1 that there exists a contraction $T_{0}$ from the span $V$ of the $x_{i}$ 's, $i=1,2, \ldots, m$ into $H$ such that $T_{0} x_{i}=y_{i}, i=1,2, \ldots, m$. We may extend $T_{0}$ to an operator $T$ on $H$ by defining $T$ to be 0 on $V^{\perp}$. Now assume that the length of $\mathscr{N}$ is $k>1$. Let $H_{0}$ be the Hilbert space $N_{1}^{\perp} H$, and let $\mathscr{N}_{0}$ be the nest in $H_{0}$ consisting 
of all projections of the form $N_{1}^{\perp} N$ with $N$ in $\mathscr{N}$. It is easy to see that if $L$ belongs to $\mathscr{N}_{0}$ then:

$$
\left\|L^{\perp}\left(\sum_{i=1}^{m} c_{i} N_{1}^{\perp} y_{i}\right)\right\| \leq M \cdot\left\|L^{\perp}\left(\sum_{i=1}^{m} c_{i} N_{1}^{\perp} x_{i}\right)\right\|,
$$

for every $m$-tuple $\left(c_{1}, c_{2}, \ldots, c_{m}\right)$ of complex numbers. Hence, by the induction hypothesis, there exists a contraction $A_{0}$ in $\operatorname{Alg} \mathscr{N}_{0}$ such that $A_{0} N_{1}^{\perp} x_{i}=$ $N_{1}^{\perp} y_{i}$ for every $i, 1 \leq i \leq m$. We denote by $T_{0}$ the operator defined by $T_{0} x=A_{0} x$ if $x \in N_{1}^{\perp} H, T_{0} x=0$ if $x \in N_{1} H$. It is easy to see that $T_{0}$ belongs to $\operatorname{Alg} \mathscr{N}$. For each $\left(c_{1}, c_{2}, \ldots, c_{m}\right)$ in $\mathbb{C}^{m}$ we have:

$$
\left\|N_{1}\left(\sum_{i=1}^{m} c_{i} y_{i}\right)\right\| \leq\left\|\sum_{i=1}^{m} c_{i} y_{i}\right\| \leq 1 \cdot\left\|\sum_{i=1}^{m} c_{i} x_{i}\right\| .
$$

Thus by Lemma 1, there exists a contraction $A_{1}: V \rightarrow H$ such that $A_{1} x_{i}=N_{1} y_{i}$ for every $i, 1 \leq i \leq m$. For each $\left(c_{1}, c_{2}, \ldots, c_{m}\right)$ in $\mathbb{C}^{m}$, we have:

$$
\begin{gathered}
\left\|N_{1}\left(\sum_{i=1}^{m} c_{i} y_{i}\right)\right\|^{2}+\left\|N_{1}^{\perp}\left(\sum_{i=1}^{m} c_{i} y_{i}\right)\right\|^{2} \leq\left\|\sum_{i=1}^{m} c_{i} x_{i}\right\|^{2} \\
\Rightarrow\left\|A_{1}\left(\sum_{i=1}^{m} c_{i} x_{i}\right)\right\|^{2} \leq\left\|\sum_{i=1}^{m} c_{i} x_{i}\right\|^{2}-\left\|N_{1}^{\perp}\left(\sum_{i=1}^{m} c_{i} y_{i}\right)\right\|^{2} \\
=\left\|\sum_{i=1}^{m} c_{i} x_{i}\right\|^{2}-\left\|N_{1}^{\perp} T_{0} N_{1}^{\perp}\left(\sum_{i=1}^{m} c_{i} x_{i}\right)\right\|^{2},
\end{gathered}
$$

since $T_{0} N_{1}^{\perp} x_{i}=N_{1}^{\perp} y_{i}$ for $1 \leq i \leq m$ by construction. It follows that for every $v$ in $V$, we have:

$$
\left\|A_{1} v\right\|^{2} \leq\|v\|^{2}-\left\|N_{1} T_{0} N_{1}^{\perp} v\right\|^{2} .
$$

Rewrite this as:

(*) $\quad\left\|A_{1} v\right\|^{2} \leq B(v, v), \quad$ where $B(x, y)=\left\langle x,\left(I-N_{1}^{\perp} T_{0}^{*} N_{1}^{\perp} T_{0} N_{1}^{\perp}\right) y\right\rangle$

for $x, y$ in $H$. Since $\left\|T_{0}\right\| \leq 1, I-N_{1}^{\perp} T_{0}^{*} N_{1}^{\perp} T_{0} N_{1}^{\perp}$ is a positive contraction. By Lemma 2, there exist a closed subspace $V_{0}$ of $H$ such that $V+V_{0}=H$, $V \cap V_{0}=\{0\}$, and $B(x, y)=0$ for every $x$ in $V$ and $y$ in $V_{0}$. We define an operator $T_{1}: H \rightarrow H$ by: $T_{1} x=A_{1} x$ if $x \in V, T_{1} x=0$ if $x \in V_{0}$.

Claim. $\left\|T_{1} x\right\|^{2} \leq B(x, x)$, for every $x$ in $H$.

Indeed, let $x$ be in $H$. Then $x$ is of the form $x=v+v_{0}$ with $v \in V$ and $v_{0} \in V_{0}$. We have:

$$
\begin{aligned}
\left\|T_{1} x\right\|^{2} & =\left\|T_{1}\left(v+v_{0}\right)\right\|^{2}=\left\|A_{1} v\right\|^{2} \leq B(v, v), \quad \text { by }(*) \\
& \leq B(v, v)+B\left(v_{0}, v_{0}\right)=B\left(v+v_{0}, v+v_{0}\right)=B(x, x) .
\end{aligned}
$$

The range of $T_{1}$ being contained in $N_{1} H, T_{1}$ belongs to $\operatorname{Alg} \mathscr{N}$. We may now define $T=T_{1}+T_{0}$. Then $T \in \operatorname{Alg} \mathscr{N}$ and $T x_{i}=y_{i}$ for every $i, 1 \leq i \leq m$. It remains to see that $T$ is a contraction. Indeed, for every $x$ in $H$, we have:

$$
\begin{aligned}
\|T x\|^{2} & =\left\|T_{1} x+T_{0} x\right\|^{2}=\left\|T_{1} x\right\|^{2}+\left\|T_{0} x\right\|^{2} \\
& \leq B(x, x)+\left\|T_{0} x\right\|^{2}=\|x\|^{2}-\left\|N_{1}^{\perp} T_{0} N_{1}^{\perp} x\right\|^{2}+\left\|T_{0} x\right\|^{2}=\|x\|^{2},
\end{aligned}
$$


where we have used the fact that $T_{0}=N_{1}^{\perp} T_{0} N_{1}^{\perp}$.

Theorem 4. Let $\mathcal{N}$ be a nest in $H$. Let $\left\{x_{\alpha}\right\},\left\{y_{\alpha}\right\}$ be two families of vectors in $H$. Let $M \geq 0$. The following are equivalent:

(1) There exist $T$ in $\operatorname{Alg} \mathscr{N}$ such that $T x_{\alpha}=y_{\alpha}$ for every $\alpha$ and $\|T\| \leq M$.

(2) $\left\|N^{\perp}\left(\sum c_{\alpha} y_{\alpha}\right)\right\| \leq M \cdot\left\|N^{\perp}\left(\sum c_{\alpha} x_{\alpha}\right)\right\|$, for every $N$ in $\mathscr{N}$ and for every family $\left\{c_{\alpha}\right\}$ of complex numbers, that is 0 except for finitely many indices.

Proof. The implication $(1) \Rightarrow(2)$ is as easy as in the finite nest case.

For the converse, first assume that the families $\left\{x_{\alpha}\right\},\left\{y_{\alpha}\right\}$ are finite.

Let $\mathscr{L}$ be the set of finite subnests of $\mathscr{N}$ directed by inclusion. It follows from the proposition that for every $\mathscr{F}$ in $\mathscr{L}$ there exists an operator $T_{\mathscr{F}}$ in $\operatorname{Alg} \mathscr{F}$ such that $T_{\mathscr{F}} x_{\alpha}=y_{\alpha}$ for every $\alpha$ and $\left\|T_{\mathscr{F}}\right\| \leq M$. The net $\left\{T_{\mathscr{F}}\right.$ : $\mathscr{F} \in \mathscr{L}\}$ is contained in the set $\{T \in B(H):\|T\| \leq M\}$, which is compact in the weak operator topology. Let $T$ be a weak operator limit of the net $\left\{T_{\mathscr{F}}: \mathscr{F} \in \mathscr{L}\right\}$. Clearly, $\|T\| \leq M$ and $T x_{\alpha}=y_{\alpha}$ for every $\alpha$. Also it is easy to see that $T$ leaves every $N$ in $\mathscr{N}$ invariant.

For the general case, we may assume that the families $\left\{x_{\alpha}\right\},\left\{y_{\alpha}\right\}$ are indexed by an infinite set $A$. Then it follows from above that for every finite subset $U$ of $A$ there exists an operator $T_{U}$ in $\operatorname{Alg} \mathscr{N}$ such that $T_{U} x_{\alpha}=y_{\alpha}$ for every $\alpha$ in $U$ and $\left\|T_{U}\right\| \leq M$. Let $T$ be a weak operator limit point of the net $\left\{T_{U}\right\}$. Then $\|T\| \leq M, T x_{\alpha}=y_{\alpha}$ for every $\alpha$ in $A$ and $T$ belongs to $\operatorname{Alg} \mathscr{N}$ since $\operatorname{Alg} \mathcal{N}$ is closed in the weak operator topology.

The following corollary generalizes Theorem 4.3 of [2].

Corollary 5. Let $A$ and $B$ be operators in $B(H)$, and let $\mathscr{N}$ be a nest in $H$. Let $M \geq 0$. The following are equivalent:

(1) There exists an operator $X$ in $\operatorname{Alg} \mathscr{N}$ such that $A=X B$ and $\|X\| \leq M$.

(2) $\left\|N^{\perp} A x\right\| \leq M \cdot\left\|N^{\perp} B x\right\|$, for every $N$ in $\mathcal{N}$ and $x$ in $H$.

Proof. Let $\left\{e_{\alpha}\right\}$ be an orthonormal basis of $H$. We set $y_{\alpha}=A e_{\alpha}, x_{\alpha}=B e_{\alpha}$ and apply the Theorem.

Remark. After this work was completed, it came to our attention (private communication with E. Katsoulis) that E. Katsoulis, R. Moore, and T. Trent have arrived at similar results.

\section{REFERENCES}

1. M. Anoussis, E. Katsoulis, R. Moore, and T. Trent, Interpolation problems for ideals in certain reflexive operator algebras, preprint.

2. W. Arveson, Interpolation problems in nest algebras, J. Funct. Anal. 20 (1975), 208-233.

3. A. Hopenwasser, The equation $T x=y$ in a reflexive operator algebra, Indiana Univ. Math. J. 29 (1980), 121-126.

4. __ Hilbert-Schmidt interpolation in CSL algebras, Illinois J. Math. 33 (1989), 657-672.

5. E. Lance, Some properties of nest algebras, Proc. London Math. Soc. (3) 19 (1969), 45-68.

Department of Mathematics, Aegean University, Karlovassi 83200 Greece 